Full Length Article

Basic Research

\title{
Mechanism for the attenuation of neutrophil and complement hyperactivity by MSC exosomes
}

\author{
Jia Tong Loh ${ }^{1}$, Bin Zhang ${ }^{2}$, Joey Kay Hui Teo ${ }^{1}$, Ruenn Chai Lai ${ }^{2}$, Andre Boon Hwa Choo ${ }^{3}$, \\ Kong-Peng Lam ${ }^{1,4,5, *}$, Sai Kiang Lim ${ }^{2,6,7, * *}$ \\ ${ }^{1}$ Singapore Immunology Network, Agency for Science, Technology and Research, Republic of Singapore \\ ${ }^{2}$ Institute of Molecular and Cell Biology, Agency for Science, Technology and Research, Republic of Singapore \\ ${ }^{3}$ Bioprocessing Technology Institute, Agency for Science, Technology and Research, Republic of Singapore \\ ${ }^{4}$ Department of Microbiology and Immunology, Yong Loo Lin School of Medicine, National University of Singapore, Republic of Singapore \\ ${ }^{5}$ School of Biological Sciences, College of Science, Nanyang Technological University, Republic of Singapore \\ ${ }^{6}$ Department of Surgery, Yong Loo Lin School of Medicine, National University of Singapore, Republic of Singapore
}

\section{A R T I C L E I N F O}

\section{Article History:}

Received 14 September 2021

Accepted 29 December 2021

\section{Keywords:}

complements

exosomes

IL-17

netosis

neutrophils

\begin{abstract}
A B S T R A C T
Complements and neutrophils are two key players of the innate immune system that are widely implicated as drivers of severe COVID-19 pathogenesis, as evident by the direct correlation of respiratory failure and mortality with elevated levels of terminal complement complex C5b-9 and neutrophils. In this study, we identified a feed-forward loop between complements and neutrophils that could amplify and perpetuate the cytokine storm seen in severe SARS-CoV-2-infected patients. We observed for the first time that the terminal complement activation complex C5b-9 directly triggered neutrophil extracellular trap (NET) release and interleukin (IL)-17 production by neutrophils. This is also the first report that the production of NETs and IL17 induced by C5b-9 assembly on neutrophils could be abrogated by mesenchymal stem cell (MSC) exosomes. Neutralizing anti-CD59 antibodies abolished this abrogation. Based on our findings, we hypothesize that MSC exosomes could alleviate the immune dysregulation in acute respiratory failure, such as that observed in severe COVID-19 patients, by inhibiting complement activation through exosomal CD59, thereby disrupting the feed-forward loop between complements and neutrophils to inhibit the amplification and perpetuation of inflammation during SARS-CoV-2 infection.

C 2022 International Society for Cell \& Gene Therapy. Published by Elsevier Inc. This is an open access article under the CC BY-NC-ND license (http://creativecommons.org/licenses/by-nc-nd/4.0/)
\end{abstract}

\section{Introduction}

The COVID-19 pandemic is a global public health emergency caused by severe acute respiratory syndrome coronavirus 2 (SARS-CoV2), affecting $>200$ million individuals to date, with a significant mortality rate of $\sim 2 \%$ (WHO, covid19.who.int). The unprecedented rapid development and rollout of vaccines have significantly reduced mortality. However, a significant number of people remain unvaccinated and are at risk

\footnotetext{
* Corresponding author. Kong-Peng Lam: Singapore Immunology Network, Agency for Science, Technology and Research, 8A Biomedical Grove, S138648, Republic of Singapore. Tel: +65 64070011

** Corresponding author. Sai Kiang Lim: Institute of Molecular and Cell Biology, Agency for Science, Technology and Research, 8A Biomedical Grove, \#05-39 Immunos, S138648, Republic of Singapore. Tel: +65 64070161 or +65 64070150

E-mail addresses: lam_kong_peng@immunol.a-star.edu.sg (K.-P. Lam), lim_sai_kiang@imcb.a-star.edu.sg (S.K. Lim).

7 Tel: +65 64070161 or +65 64070150
}

of developing severe disease. Severe COVID-19 disease is essentially a manifestation of dysregulated immune responses in the lungs that could lead to multi-organ pathologies. Current COVID-19 interventions are generally symptomatic and palliative treatments, and there is an urgent unmet need for effective drugs to alleviate immune dysfunctions in the lungs of severely ill COVID-19 patients to reduce mortality and improve recovery.

Mesenchymal stromal cell (MSC)-derived exosomes, which have documented immunomodulatory and regenerative properties, have been proposed as a promising therapeutic to alleviate immune dysfunction and reduce disease severity in fatally ill COVID-19 patients [1]. Although several preclinical and clinical studies have demonstrated their safety and efficacy in mitigating the symptoms associated with severe COVID19 [2,3], the precise mechanism by which MSC exosomes exert their effects to benefit COVID-19 patients remains elusive.

Our innate immune system serves as the first line of defense against viral invasion. However, an augmented or dysregulated 
innate immune response could exacerbate the pathogenesis of a viral infection such as the development of acute respiratory distress syndrome (ARDS) and cytokine storm syndrome in severe COVID-19 [4-7]. Complement is an integral component of the innate immune system that eliminates viral pathogens directly and indirectly through opsonization, formation of the membrane attack complex (MAC) C5b-9 and recruitment of other leukocytes to promote an anti-viral inflammatory response [8,9]. However, clinical data have revealed that increased activation of the complement system is a distinct immunologic feature correlating with worse COVID-19 outcomes [10-17]. Elevated levels of circulating complement markers were present in patients with COVID-19 compared with other etiologies of acute respiratory failure, and these markers can distinguish those with worse outcomes in the setting of SARS-CoV-2 infection [10]. Indeed, complement activation has been known to play a central role in endothelial injury and hypercoagulability, leading to multiorgan failure and pathogenesis of COVID-19. As such, complement inhibitors to combat SARS-CoV-2 infection are currently being explored in multiple phase II and III clinical trials [18-21].

Another immunologic indicator of poor disease outcome in COVID19 is the increased number of circulating neutrophils. In critically ill patients, extensive neutrophil extracellular traps (NETs), a prominent signature of neutrophil activation, are detected in sera and lungs, thereby predisposing the patients to thrombosis, organ damage and, eventually, death [22-28]. This is further exacerbated by the hyperactivation of complements in the lungs of severely ill COVID-19 patients [2], as activated complements are potent chemoattractants for neutrophils $[29,30]$. Together, the clinical data suggest that the cardinal roles of complements and neutrophils in the pathogenesis of severe COVID19 intersect. Therefore, elucidating and modulating this intersection represents a potential strategy to prevent the devastating complications in SARS-CoV-2-infected patients [31,32].

In this study, we show that the terminal complement activation complex C5b-9 can directly drive the release of NETs and interleukin (IL)17 by neutrophils. MSC exosomes can suppress this complement-mediated neutrophil activation via a CD59-dependent mechanism. Together, our data revealed a novel cross-talk between two innate immune components, complement and neutrophil, and further highlighted the potency of MSC exosomes in suppressing the activation of this innate immune axis. Our data also provide a compelling rationale for the use of MSC exosome to treat severe COVID-19.

\section{Results}

Terminal complement complex triggers NET formation and IL-17 release by neutrophils

To characterize the effects of complement activation on neutrophil responses, we purified neutrophils from the blood of healthy human donors and initiated complement activation using a standard protocol of adding complements C5b- 6 and C7 followed by C8 and C9. The assembly of terminal complement complex C5b-9 on neutrophil surfaces was confirmed by flow cytometry (Figure 1A). Complement activation triggered the release of NETs in vitro, as visualized microscopically via the colocalization of DAPI-positive extracellular DNA fibers with citrullinated histone $\mathrm{H} 3$. In particular, $>60 \%$ of neutrophils underwent NETosis upon activation of the complement cascade, as determined by DNA size, and the area of released NETs was significantly larger than that from the control group (Figure 1B, C). As the severity of COVID-19 also correlates positively with the level of neutrophil-associated inflammatory mediators such as IL-17 and calprotectin S100A8 and S100A9 [33-38], we determined whether these inflammatory mediators were induced in the neutrophil response to complement activation. Quantitative reverse-transcription real-time polymerase chain reaction (PCR) and ELISA analysis revealed a significant induction of IL-17 transcription and secretion upon complement activation (Figure 1D, E), while levels of s100a8 and $s 100 a 9$ transcripts remain unchanged (Figure 1F). Together, these data showed that complement activation can specifically induce NETs and IL-17 production by neutrophils. These data also provided a possible mechanistic link between complements and neutrophils to induce hyperinflammatory immune responses in severely ill COVID19 patients.

\section{Exosomes inhibit complement-induced neutrophil activation}

MSC exosomes, which possess immunomodulatory properties, have been proposed as a therapeutic option for COVID-19 to alleviate complications such as acute respiratory distress syndrome $[2,3,39]$. However, the mechanism by which exosomes exert their functions remains unclear. To test whether MSC exosomes can intervene in complement-induced neutrophilic responses, we incubated neutrophils with exosomes and complements C5b-6 and C7 followed by C8 and C9. Activation of neutrophils was attenuated upon addition of exosomes. Specifically, exosomes abrogated the release of NETs by neutrophils at concentration as low as $1 \mu \mathrm{g} / \mathrm{mL}$ (Figure 2A, B). Exosomes also inhibited IL-17 production at the transcript and protein levels by C5b-9-assembled neutrophils at 10 and $50 \mu \mathrm{g} / \mathrm{mL}$ (Figure 2C, D). The inhibition was statistically significant at 10 but not $1 \mu \mathrm{g} / \mathrm{mL}$ exosome, indicating a dose-dependent response. However, the level of inhibition at 10 and $50 \mu \mathrm{g} / \mathrm{mL}$ was not significantly different, suggesting that maximum inhibition was reached at $10 \mu \mathrm{g} / \mathrm{mL}$. Therefore, MSC exosomes can attenuate the complement-mediated NET formation and neutrophil IL-17 production.

\section{Exosomes do not affect PMA-induced NETosis and IL-17 release}

Several stimuli are known to trigger NET formation and IL-17 production by neutrophils. To determine whether inhibition of NETosis and IL-17 release by exosomes is specific to complement-mediated activation of neutrophils, we treated neutrophils with another known neutrophil activator, phorbol myristate acetate (PMA), which activates neutrophils through the signal transduction enzyme protein kinase C [40]. PMA induced NETosis and IL-17 production at the transcript and protein levels in neutrophils, but MSC exosomes did not have any effect on these processes (Figure 3A-D). Therefore, exosomes suppressed complement but not PMA-mediated activation of neutrophils. This suggested that exosomes exert their inhibition of complement-mediated neutrophil activation through the complement cascade and not through the neutrophil per se.

\section{MSC exosomes inhibit complement-induced neutrophil activation through a CD59-dependent mechanism}

MSC exosomes have been reported to inhibit the formation of the terminal complement complex, C5b-9, through their expression of CD59 [41]. To investigate whether exosomal CD59 is required for exosome-mediated suppression of complement-induced neutrophil activation, a neutralizing antibody against CD59 was added to the mixture of activated complements and neutrophils. The anti-CD59 antibody abrogated the inhibitory effects of exosomes on complement-induced NETosis. Notably, the NETs released by neutrophils in the presence of exosomes and the anti-CD59 antibody were of comparable size to those found in the control group without exosome treatment (Figure 4A, B). Consistent with this, the inhibition of IL-17 release by exosomes was also abolished by anti-CD59 antibody treatment (Figure 4C, D). Of note, anti-CD59 antibody alone does not trigger the release of NETs or IL-17, ruling out nonspecific effects of antiCD59 antibody on neutrophil activation (Figure 4A-D). Together, these results indicate that inhibition of C5b-9 formation by CD59 on the MSC exosome abolished complement-induced neutrophil NETosis and IL-17. 
A

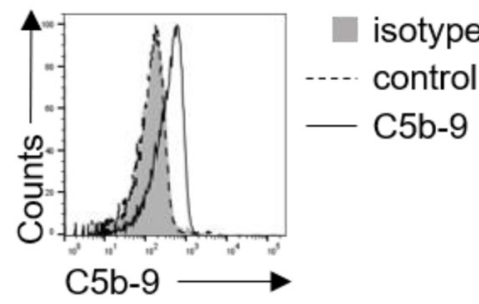

C

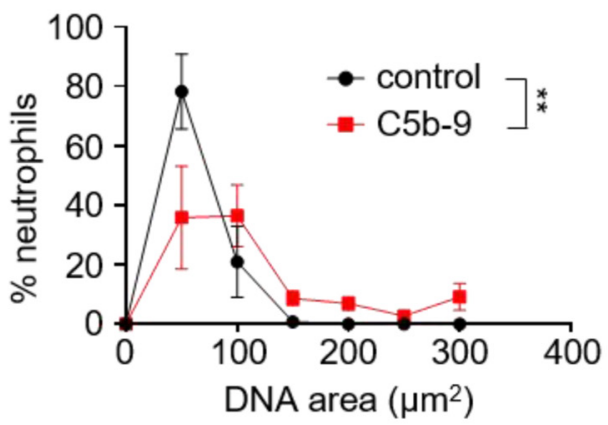

$\mathrm{D}$

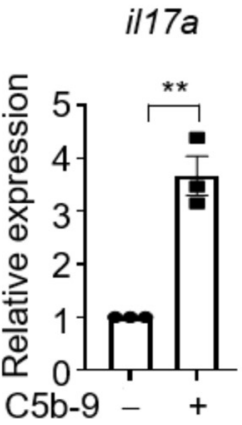

E

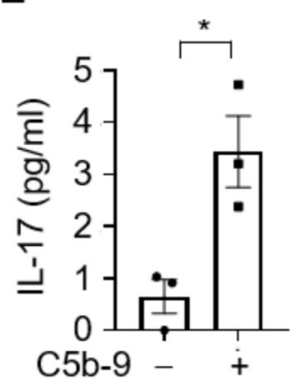

B
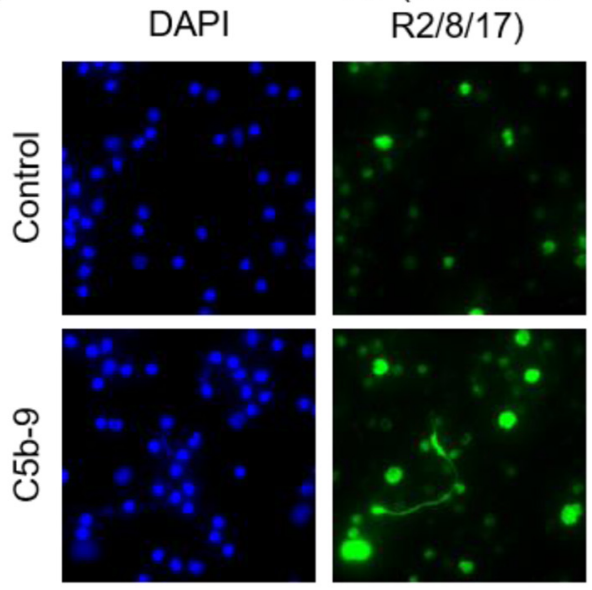
$\mathrm{R} 2 / 8 / 17)$

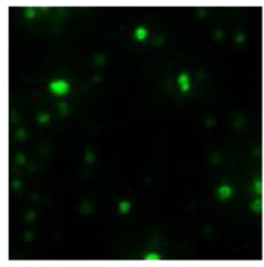

$\mathrm{H} 3$ (citrullinate

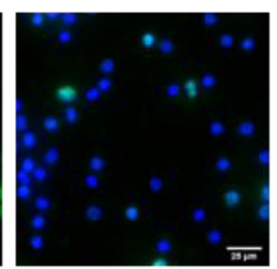

$\mathrm{F}$
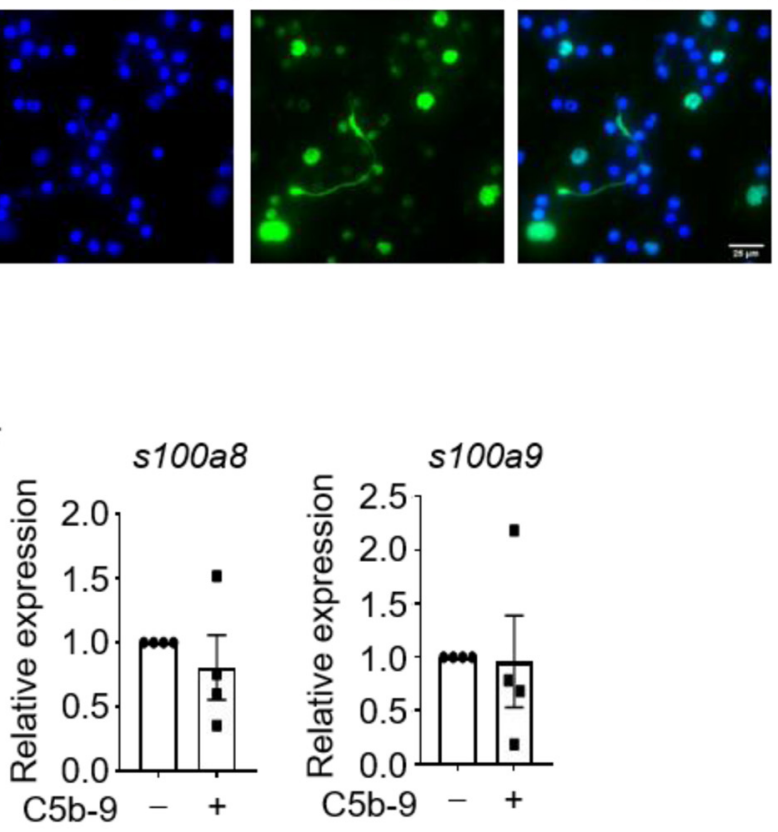

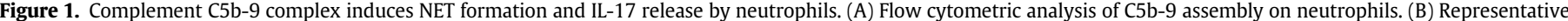

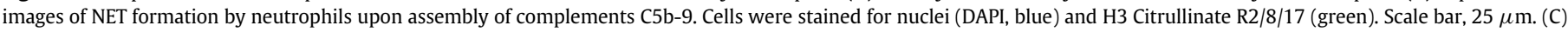

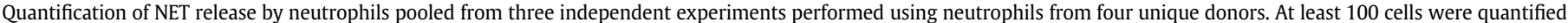

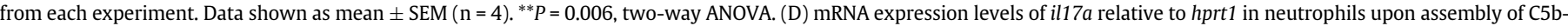

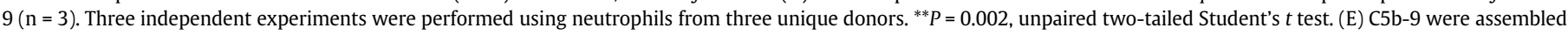

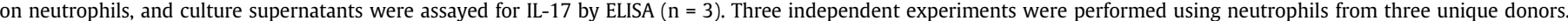

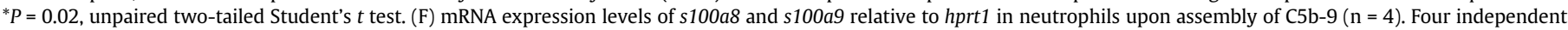
experiments were performed using neutrophils from four unique donors. (Color version of figure is available online.)

\section{Discussion}

Hyperactivation of the complement system and neutrophilic immune responses have been associated with worse outcomes in COVID-19 patients [10-12,16,23,28,35,42]. It remains unclear, however, whether these are two independent events or are interrelated components of a broader immune cascade. In this study, we demonstrated that initiation of the complement activation cascade induced NET release and IL-17 production by neutrophils. These neutrophilic responses were suppressed by MSC exosomes via a CD59-dependent mechanism (Figure 5). Because CD59 inhibits only the terminal step of the complement activation cascade, i.e., C5b-9 complex, our data also demonstrated that $\mathrm{C} 5 \mathrm{~b}-9$, and not the other complement complexes, was the main effector of neutrophil activation. Collectively, our results provide a mechanistic basis for how exosomes can inhibit complement-induced neutrophil activation, potentially mitigating the vicious cycle that perpetuates inflammation in the lungs of severely ill COVID-19 patients.

Clinical observations strongly indicate that patients with severe COVID-19 are predisposed to complement activation and dysregulated neutrophilic responses [24,25,43]. Interestingly, it has been reported that the sera from COVID-19 patients can trigger the release of NETs by healthy control neutrophils [24,44], consistent with a possibility that complements present in the sera can activate neutrophils
[45]. In this study, we present novel findings on the cross-talk between the terminal complement complex and neutrophils. We showed that the assembly of complement complexes can directly trigger NET formation and IL-17 release by neutrophils. NETs are known to fuel pro-inflammatory immune responses, and their aggregation culminates in vascular occlusion and organ damage, whereas IL-17 has been reported to exacerbate inflammation and destruction of the lung parenchyma $[37,46,47]$. Hence, it is likely that the complement system and neutrophils form a feed-forward loop to escalate inflammation, tissue injury and thrombosis that, if left unchecked, would lead to coagulopathy, respiratory failure and other devasting complications observed in COVID-19.

MSC exosomes are an attractive cell-free therapeutic option to treat and manage symptoms associated with COVID-19, and they have demonstrated promising results toward alleviation of major COVID-19 complications such as cytokine storm and ARDS in a small but growing number of clinical trials [2,3]. The rationale for such a treatment approach was predicated on the superior regenerative and immunomodulatory capacity of MSC exosomes [3]. Exosomes have been shown to secrete anti-inflammatory cytokines that interact with immune cells such as T cells, B cells and macrophages, thereby preventing overactivation of the immune system. Furthermore, they can deliver various growth factors that help initiate tissue repair and regeneration during lung injury [48]. Together, these studies support 
A
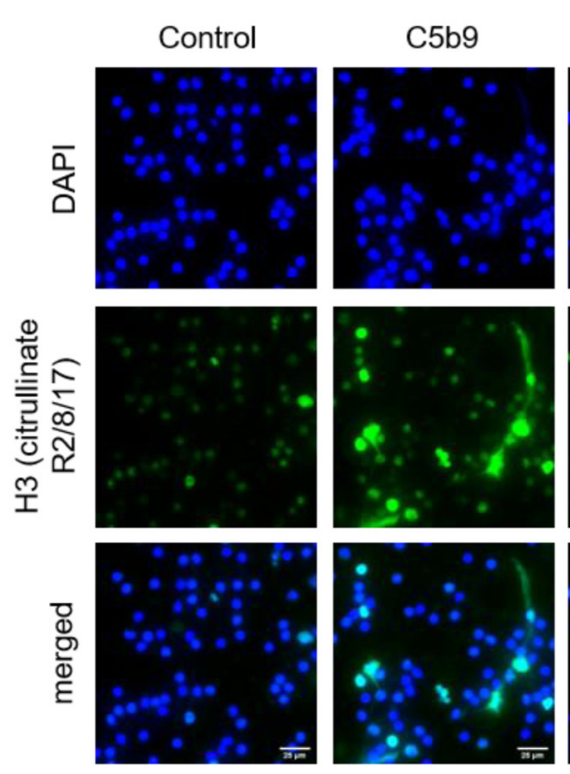

$\mathrm{C} 5 \mathrm{~b} 9+$ $1 \mu \mathrm{g} / \mathrm{ml}$ exo
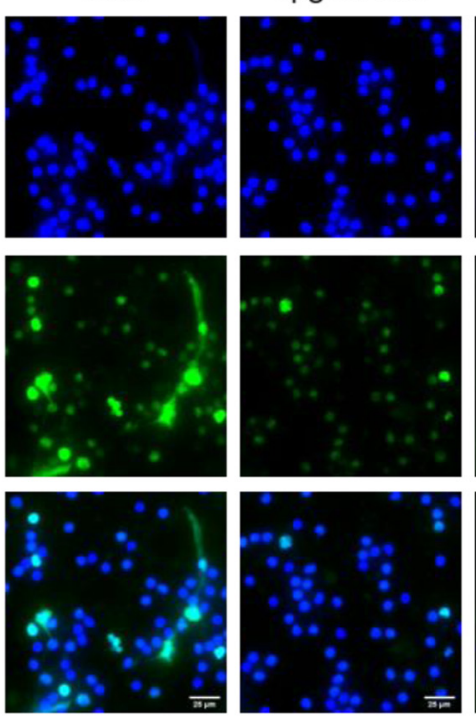

B

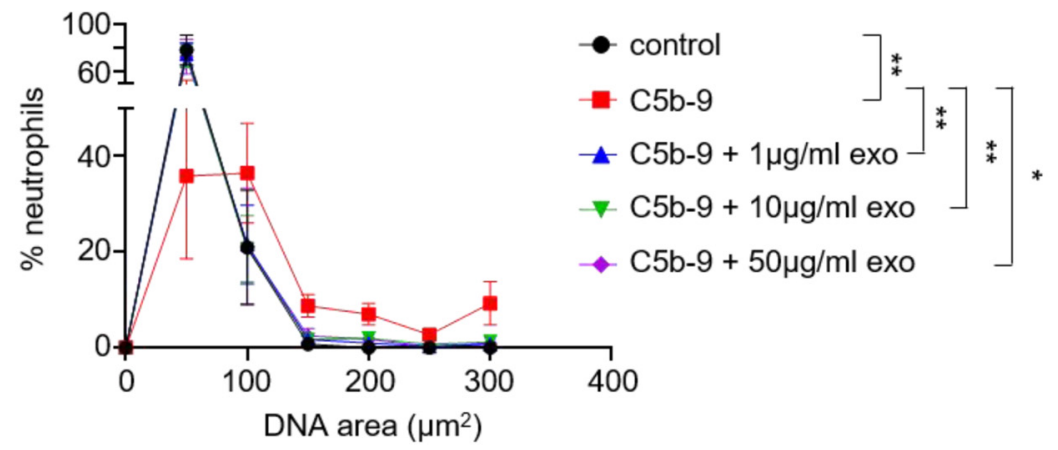

C
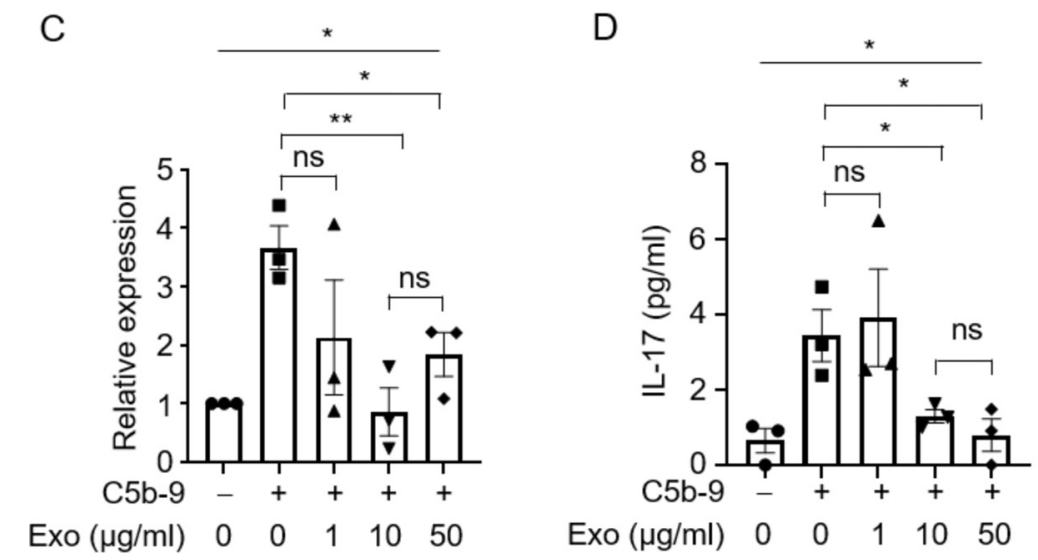

C5b9+ $10 \mu \mathrm{g} / \mathrm{ml}$ exo
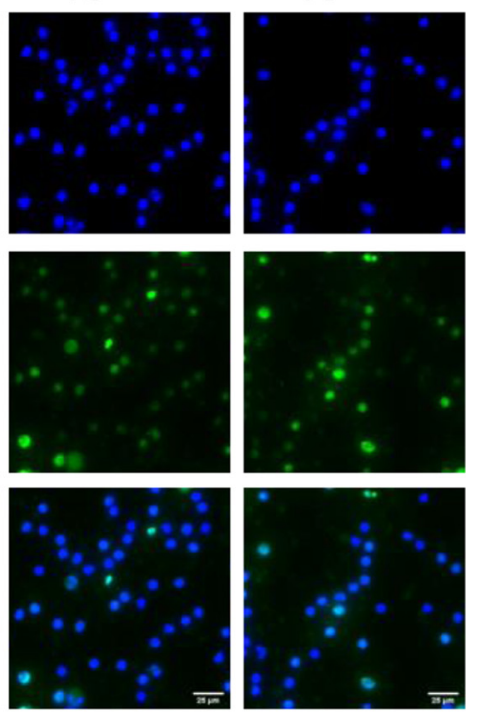

$\mathrm{C} 5 \mathrm{~b} 9+$ $50 \mu \mathrm{g} / \mathrm{ml}$ exo
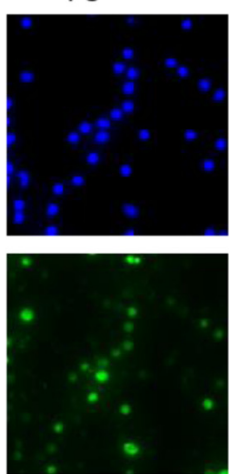

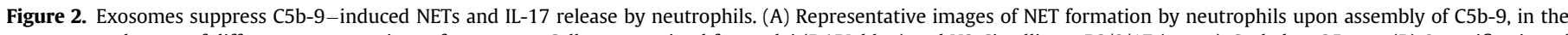

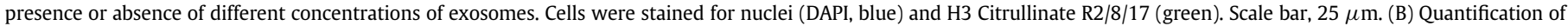

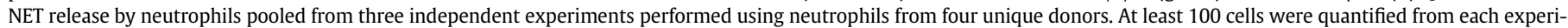

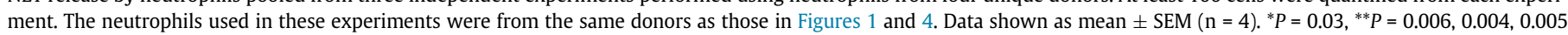

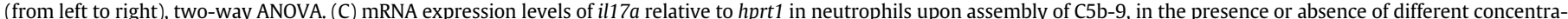

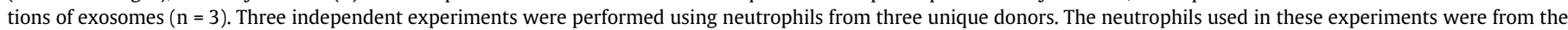

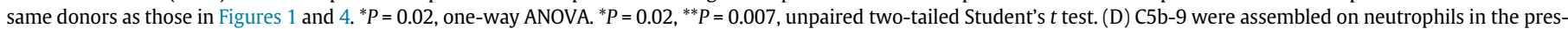

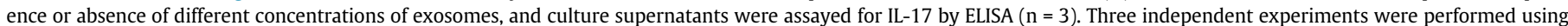

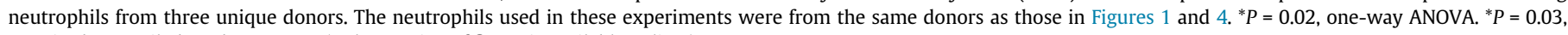
unpaired two-tailed Student's $t$ test. (Color version of figure is available online.)

the potential utility of exosomes as a treatment option for COVID-19 patients with severe complications. However, the precise therapeutic effect of exosomes on COVID-19 patients, and the exosome attribute responsible for mediating such an effect, have not been elucidated.
Here, we demonstrate for the first time that exosomes suppress complement-mediated neutrophil activation and inhibit the release of NETs and IL-17 by neutrophils (Figure 5). We identify CD59 as the MSC exosome attribute that mediates this suppression. We 
A
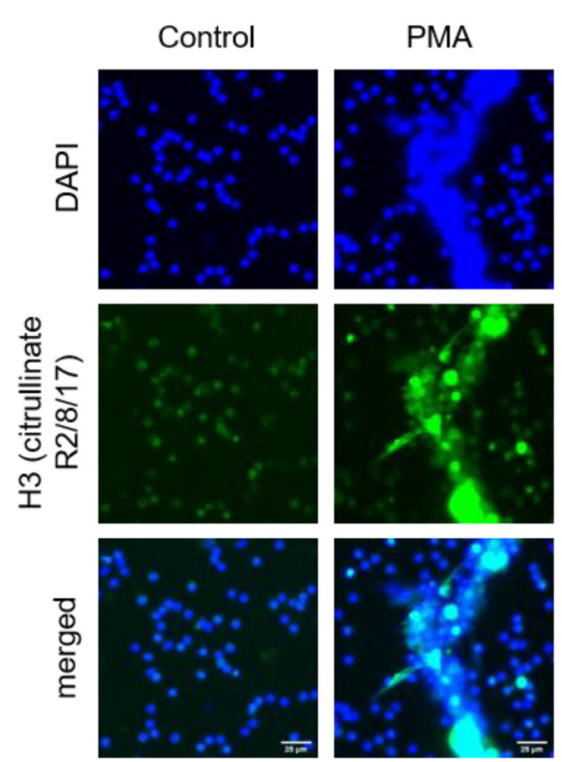

PMA + $1 \mu \mathrm{g} / \mathrm{ml}$ exo

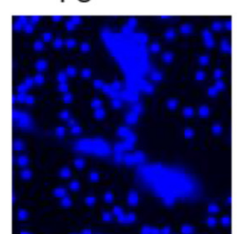

PMA + $10 \mu \mathrm{g} / \mathrm{ml}$ exo
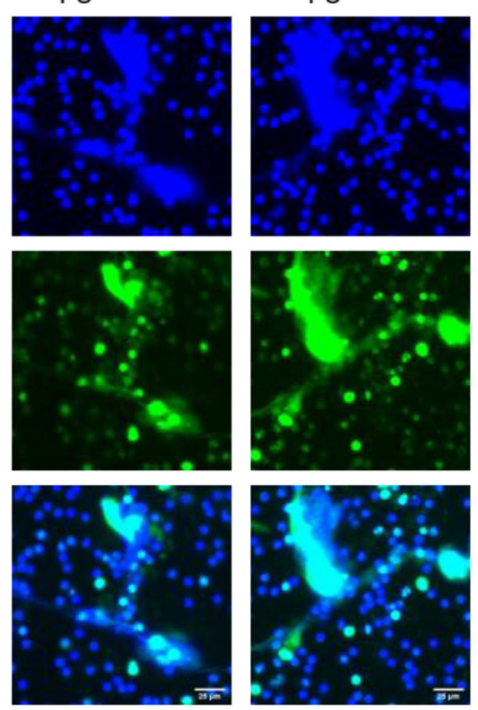

PMA + $50 \mu \mathrm{g} / \mathrm{ml}$ exo
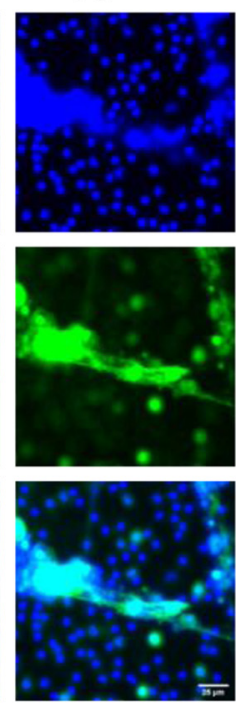

B

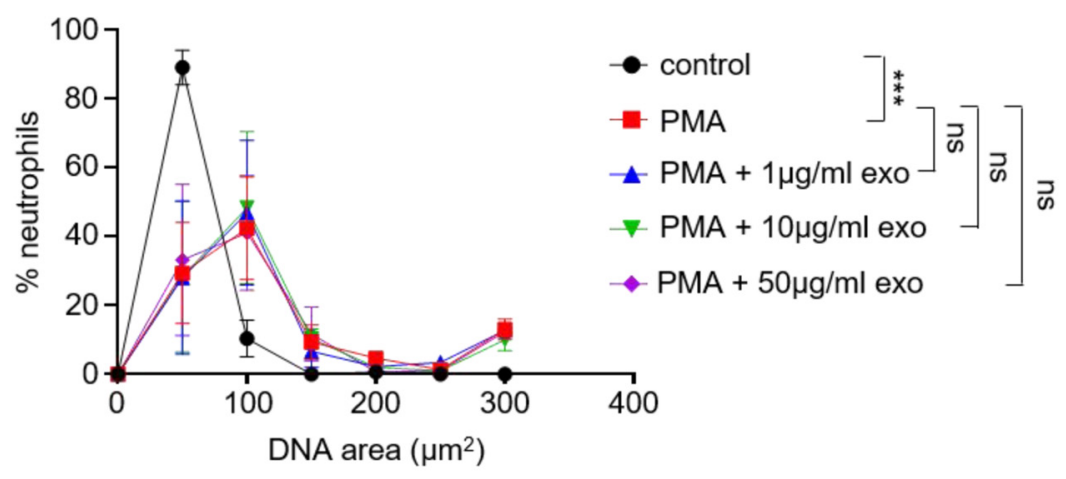

C

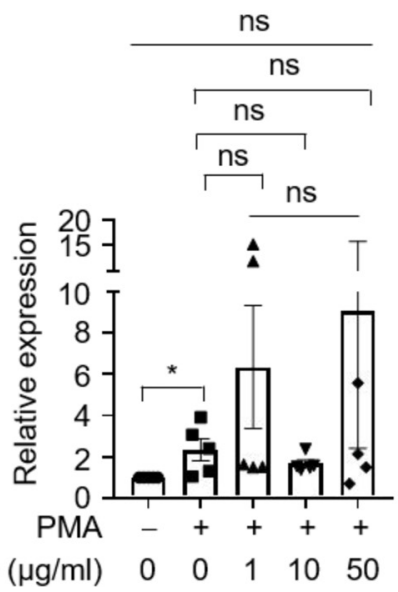

D

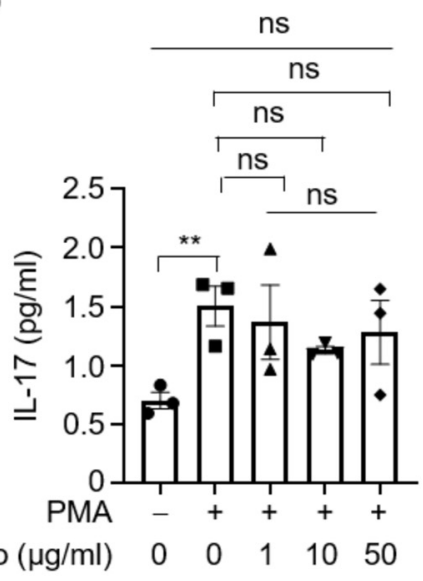

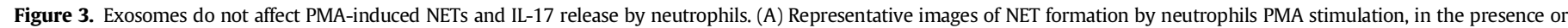

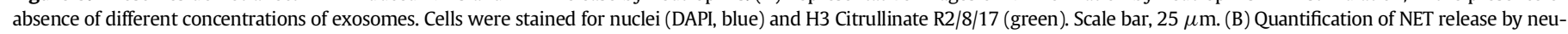

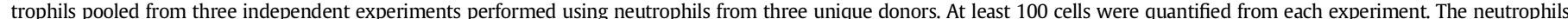

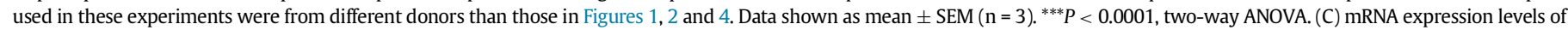

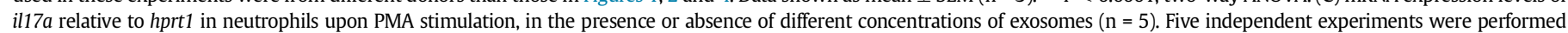

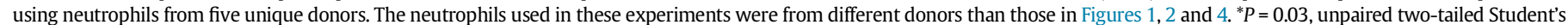

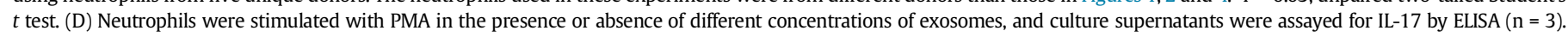

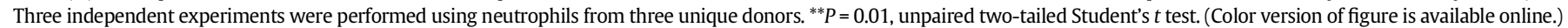

previously showed that MSC exosomes inhibit the assembly of terminal C5b-9 complement complex through CD59, a known inhibitor of the final stage in the complement activation cascade and an abundant
MSC exosomal protein [41]. Consistent with the role of CD59 in mediating the inhibitory effect of MSC exosomes on neutrophil activation, this inhibitory effect was attenuated by a CD59-neutralizing antibody 
A
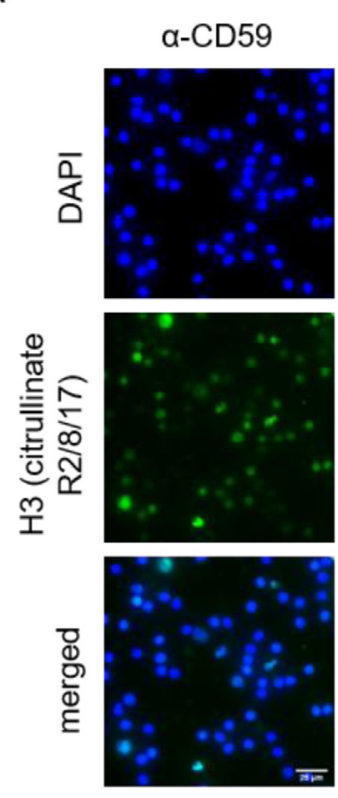

C5b9+

$10 \mu \mathrm{g} / \mathrm{ml}$ exo

$+\alpha-C D 59$
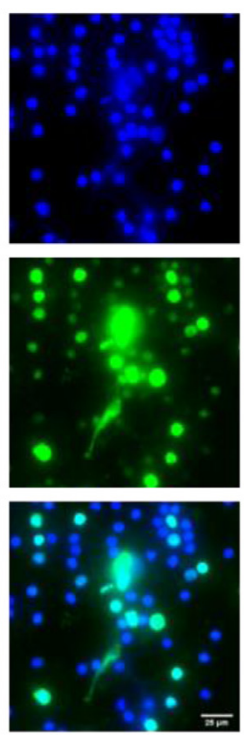

C5b9+

$50 \mu \mathrm{g} / \mathrm{ml}$ exo

+ a-CD59
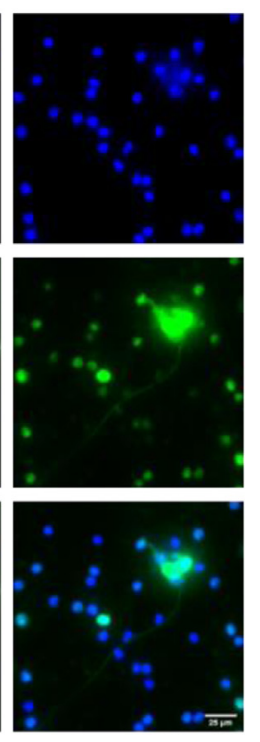

B

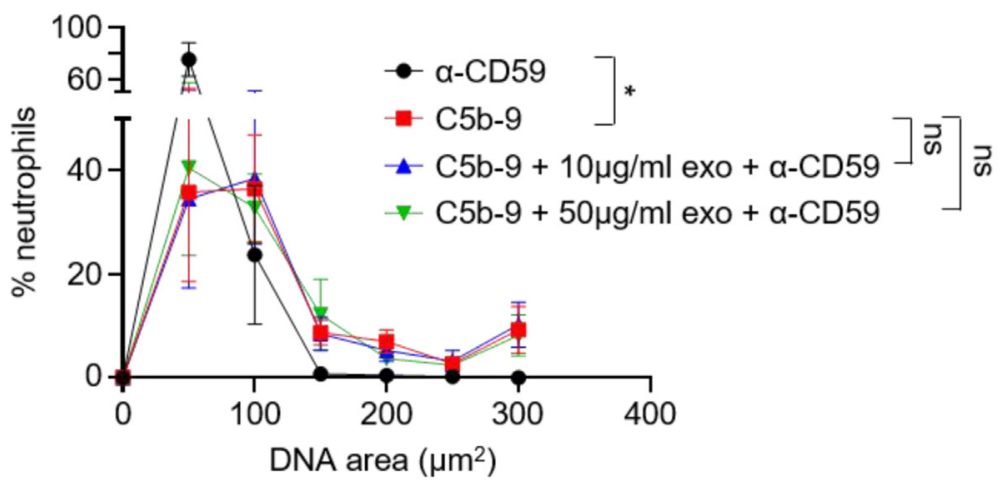

C

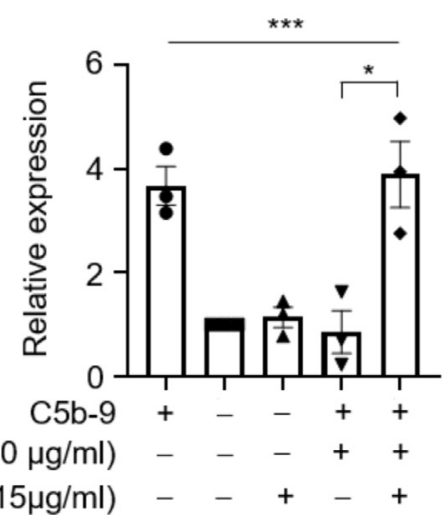

D

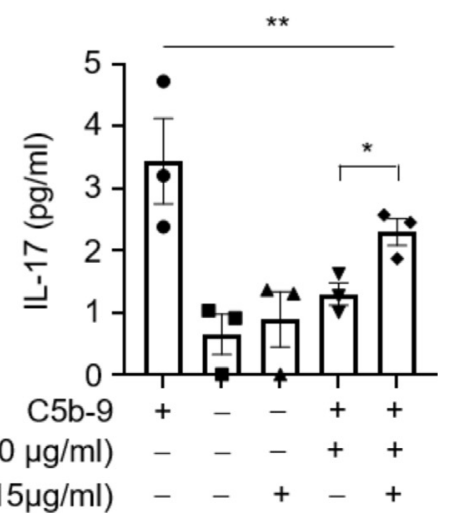

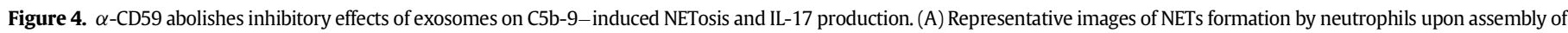

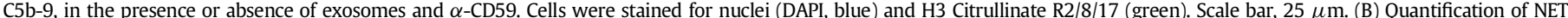

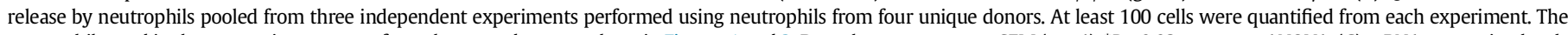

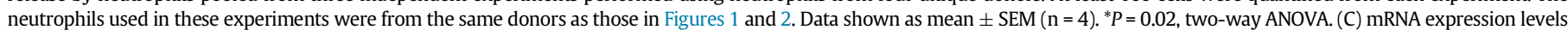

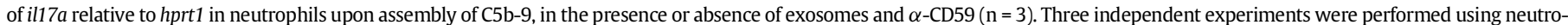

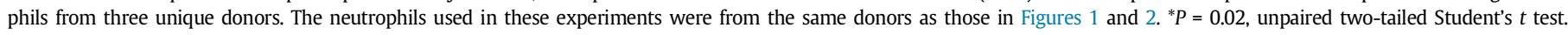

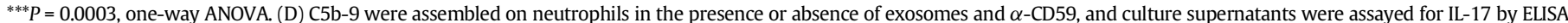

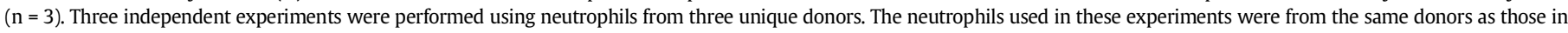
Figures 1 and $2 .{ }^{*} P=0.02$, unpaired two-tailed Student's $t$ test. ${ }^{*} P=0.004$, one-way ANOVA. (Color version of figure is available online.)

[41]. By inhibiting the formation of the terminal complement complex on neutrophils through CD59, MSC exosomes attenuated the activation of neutrophils and the manifestations of neutrophil activation, namely NETosis and IL-17 production. Significantly, we did not observe any inhibitory effect of exosomes on PMA-induced NETs and IL-17 release, indicating that MSC exosomes act specifically 


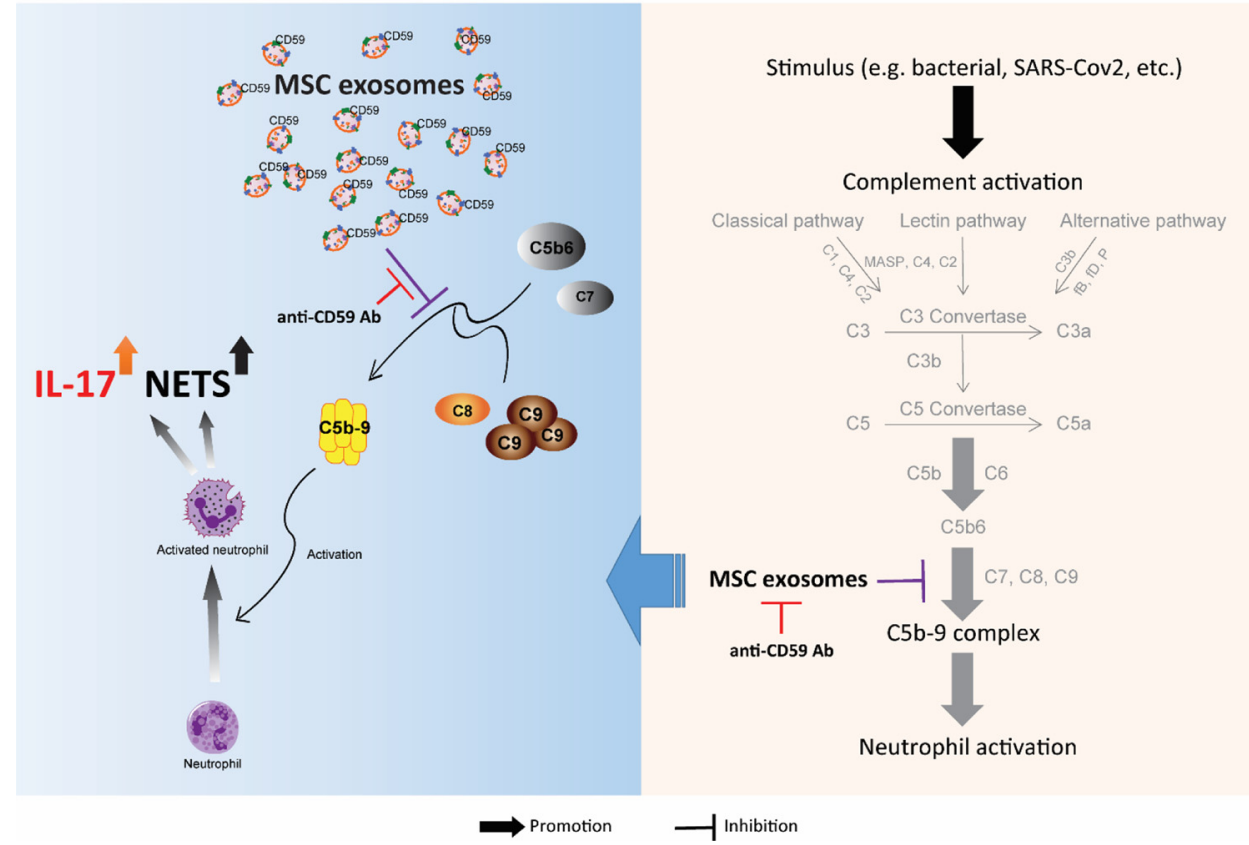

Figure 5. Schematic diagram showing MSC exosome inhibition of complement/neutrophil axis through the expression of CD59. (Color version of figure is available online.)

on complement-mediated activation of neutrophils. Together, these results also revealed for the first time that $C 5 b-9$, and not the other complement complex upstream of C5b-9, is the major complement complex that activates neutrophils.

In conclusion, our study elucidates a feed-forward loop between two key immunologic indicators of severe COVID-19, complements and neutrophils, and provides an overarching mechanistic context for the association of elevated complement activation, neutrophils, NETosis and IL-17 with adverse COVID-19 outcomes. This study also determines that MSC exosomes can specifically inhibit this complement/neutrophil axis through their expression of CD59 and provides a scientific rationale for use of MSC exosomes in alleviating the severe manifestations of critically ill COVID-19 patients.

\section{Materials and Methods}

\section{Preparation of MSC exosomes}

The exosome preparation used in this study was batch AC109 from an ongoing production process that has been in operation for $>10$ years and has manufactured $>100$ batches to date. This process was first described in 2010 [1]. The cell source was replaced with the immortalized cell line in 2013 [5], and the process was simplified as described below. Immortalized E1-MYC 16.3 human ESC-derived mesenchymal stem cells were cultured in DMEM with $10 \%$ fetal calf serum as previously described [49]. For MSC exosome preparation, the conditioned medium was prepared by growing $80 \%$ confluent cells in a chemically defined medium for 3 days as previously described [50-52]. The defined medium was prepared as follows: 480 mL DMEM (31053; Thermo Fisher), 5 mL NEAA (11140-050; Thermo Fisher), $5 \mathrm{~mL}$ L-glutamine (25030-081; Thermo Fisher), $5 \mathrm{~mL}$ sodium pyruvate (11360; Thermo Fisher), $5 \mathrm{~mL}$ ITS-X (51500-056; Thermo Fisher) and $0.5 \mathrm{~mL}$ 2-mercaptoethanol (21985-02; Thermo Fisher). This was supplemented with $0.1 \mathrm{~mL}$ basic fibroblast growth factor (bFGF; $0.5 \mathrm{ng} / \mu \mathrm{L} 0.2 \% \mathrm{BSA}$ in $\mathrm{PBS}(+)$ ) and $0.005 \mathrm{~mL}$ plateletderived growth factor (PDGF; $100 \mathrm{ng} / \mu \mathrm{L} \mathrm{PBS}(+))$. The latter components were obtained as follows: bovine serum albumin (BSA; A9647; Sigma-Aldrich), PDGF (100-00; CYTOLAB), bFGF (13256-029; Thermo Fisher) and PBS(+) (14040-133; Thermo Fisher). The conditioned medium (CM) was size-fractionated by tangential flow filtration and then concentrated $50 \times$ using a membrane with a molecular weight cutoff (MWCO) of $100 \mathrm{kDa}$ (Sartorius, Gottingen, Germany). The MSC exosome preparation was assayed for protein concentration using a Coomassie Plus (Bradford) Assay Kit (Thermo Fisher). The properties of these exosome preparations have been extensively assayed in accordance with Minimum Information for the Study of Extracellular Vesicles 2018 [6] and reported in our earlier papers. These preparations carry exosome-associated markers CD81, ALIX and TSG101 $[50,53]$, and the presence of CD81 on bi-lipid membrane vesicles was confirmed using transmission electron microscopy [54]. For this study, AC109 was assayed for the key identity and potency parameters for MSC-sEV preparations as recommended [7,8]. Specifically, the preparation had a protein concentration of $1.419 \pm 0.034 \mathrm{mg} / \mathrm{mL}$, particle concentration of $2.13 \times 10^{11} \pm 5 \times 10^{9}$ particles $/ \mathrm{mL}$, particles with a modal size of $125.1 \pm 6.0 \mathrm{~nm}$, cholesterol of $8.16 \pm 0.32 \mathrm{ng}$ cholesterol $/ \mu$ g protein, CD73/ecto-5'-nucleotidase activity of $27.04 \pm$ $0.79 \mathrm{mU} / \mu \mathrm{g}$ protein and CD59 concentration of $3.15 \pm 0.43 \mathrm{ng}$ CD59/ $\mathrm{mg}$ protein. The exosome preparation was filtered with a $0.22-\mu \mathrm{m}$ filter (Merck Millipore, Billerica, MA) and stored in a $-80^{\circ} \mathrm{C}$ freezer until use or lyophilized.

\section{Isolation of neutrophils}

Venous blood of healthy human donors was collected and diluted 1:1 in PBS before overlaying on Ficoll-Paque (GE Healthcare). After density gradient centrifugation, the polymorphonuclear and erythrocyte-rich pellet was collected, and cells were treated with red blood cell lysis buffer for $15 \mathrm{~min}$ at room temperature. Cells were subsequently washed with $\mathrm{PBS}$, and neutrophils obtained were resuspended in $\mathrm{PBS}$ and rested at $37^{\circ} \mathrm{C}$ for $\geq 1 \mathrm{~h}$.

\section{Assembly of C5b-9}

Neutrophils were washed and then resuspended in PBS with C5b$6(0.125 \mu \mathrm{g} / \mathrm{mL})$ and $\mathrm{C} 7(0.5 \mu \mathrm{g} / \mathrm{mL})$ in the presence or absence of exosomes. The mixture was incubated at $37^{\circ} \mathrm{C}$ for $15 \mathrm{~min}$ before $\mathrm{C} 8$ $(0.5 \mu \mathrm{g} / \mathrm{mL})$ and $\mathrm{C} 9(0.5 \mu \mathrm{g} / \mathrm{mL})$ were added with or without a CD59 blocking antibody (YTH53.1; Invitrogen) at $37^{\circ} \mathrm{C}$ for an additional 30 min incubation. As a negative control, $\mathrm{C} 9$ was omitted. 
Flow cytometry staining for C5b-9

Neutrophils were stained with anti-C5b-9 (aE11; Novus Biologicals), followed by anti-mouse FITC (Sigma-Aldrich), in staining buffer (PBS containing $1 \% \mathrm{BSA}$ ) at $4^{\circ} \mathrm{C}$ for $1 \mathrm{~h}$. Data were acquired using LSRII (BD Biosciences) and analyzed using FlowJo software (TreeStar).

\section{PMA stimulation}

Neutrophils were stimulated with $10 \mathrm{ng} / \mathrm{mL}$ PMA, in the presence or absence of exosomes, for $30 \mathrm{~min}$ at $37^{\circ} \mathrm{C}$.

\section{NETosis assay}

Neutrophils were seeded onto poly-L-lysine-coated 48-well plates and allowed to adhere for $30 \mathrm{~min}$ at $37^{\circ} \mathrm{C}$. The cells were then stimulated with complement components or PMA as described above. Cells were then fixed in $2 \%$ paraformaldehyde at room temperature for $15 \mathrm{~min}$ and stained with anti-histone $\mathrm{H} 3$ (citrulline R2 +R8+R17) antibody (ab5103; Abcam) and DAPI before visualization with a microscope (EVOS). DNA area was measured using DAPI staining in Image software. Image acquisition and analysis were performed by a blinded investigator. At least 100 cells in each experimental group were analyzed.

\section{IL-17 ELISA}

$2 \times 10^{6}$ neutrophils were stimulated with C5b-9 or PMA as described above. Culture supernatants were collected after $4 \mathrm{~h}$, and IL-17 was detected by human IL-17A ELISA kit (ab216167; Abcam) according to the manufacturer's protocol.

\section{Quantitative PCR}

Purified neutrophils were lysed with TRIzol (Gibco, Thermo Fisher), and RNA was purified using phenol/chloroform extraction. Complementary DNA was reversed transcribed using RevertAid First Strand cDNA Synthesis Kit (Thermo Fisher). The following primers were used for real-time PCR using SYBR Green PCR Master Mix (Applied Biosystems):

\section{Il17a (forward): TCCCACGAAATCCAGGATGC}

Il17a (reverse): GGATGTTCAGGTTGACCATCAC

s100a8 (forward): GGGCATCATGTTGACCGAGC

s100a8 (reverse): GTAACTCAGCTACTCTTTGTGGCTT

s100a9 (forward): CGATGACTTGCAAAATGTCGCAG

s100a9 (reverse): GCCACTGTGGTCTTAGGGGGT

hprt1 (forward): GAAAAGGACCCCACGAAGTGT

hprt1 (reverse): AGTCAAGGGCATATCCTACAACA

The mRNA expression of $i l 17 a$, s100a8 and s100a9 were normalized to hprt1 using the $2^{-\Delta \Delta \mathrm{Ct}}$ method.

\section{Statistics}

Figures and statistical analyses were generated using GraphPad Prism software. For NETosis analyses, two-way ANOVA was performed. For other analyses, 1-way ANOVA or unpaired two-tailed Student's $t$ test was performed. A $P$ value of $<0.05$ was considered significant.

\section{Study approval}

Human blood was obtained for research with approval from the Centralised Institutional Research Board of the Singapore Health Services in Singapore.

\section{Funding}

This work was funded by institutional core funds from A*STAR and IAF-PP (H19H6a0026, TEx2Pharm) to SKL and KPL.

\section{Author Contributions}

JTL, BZ, KPL and SKL were involved in the conceptualization and design of study. JTL and JKHT performed the experiments. ABHC produced the MSC exosomes, which were characterized by RCL. JTL, BZ, KPL and SKL analyzed and interpreted the data. The manuscript was drafted by JTL and revised by BZ, KPL and SKL. All authors have approved the final article.

\section{Declaration of Competing Interests}

SKL holds founding shares in Paracrine Therapeutics.

\section{Acknowledgments}

Human PBMCs, from which neutrophils were isolated, are provided by Singapore Health Science Authority (HSA) under project no: 201306-05 to KPL (SIgN).

\section{References}

[1] Börger V, Weiss DJ, Anderson JD, Borràs FE, Bussolati B, Carter DRF, et al. International Society for Extracellular Vesicles and International Society for Cell and Gene Therapy statement on extracellular vesicles from mesenchymal stromal cells and other cells: considerations for potential therapeutic agents to suppress coronavirus disease-19. Cytotherapy 2020;22:482-5. https://doi.org/10.1016/j. jcyt.2020.05.002.

[2] Gupta A, Kashte S, Gupta M, Rodriguez HC, Gautam SS, Kadam S. Mesenchymal stem cells and exosome therapy for COVID-19: current status and future perspective. Human Cell 2020;33:907-18. https://doi.org/10.1007/s13577-020-00407-w.

[3] Mazini L, Rochette L, Malka G. Exosomes contribution in COVID-19 patients' treatment. Journal of Translational Medicine 2021;19:234. https://doi.org/10.1186/ s12967-021-02884-5.

[4] Leisman DE, Ronner L, Pinotti R, Taylor MD, Sinha P, Calfee CS, et al. Cytokine elevation in severe and critical COVID-19: a rapid systematic review, meta-analysis, and comparison with other inflammatory syndromes. The Lancet Respiratory Medicine 2020;8:1233-44. https://doi.org/10.1016/S2213-2600(20)30404-5.

[5] Webb BJ, Peltan ID, Jensen P, Hoda D, Hunter B, Silver A, et al. Clinical criteria for COVID-19-associated hyperinflammatory syndrome: a cohort study. The Lancet Rheumatology 2020;2:e754-63. https://doi.org/10.1016/S2665-9913(20)30343-X.

[6] Chen LYC, Quach TTT. COVID-19 cytokine storm syndrome: a threshold concept. The Lancet Microbe 2021;2:e49-50. https://doi.org/10.1016/S2666-5247(20) 30223-8

[7] Wilson JG, Simpson LJ, Ferreira A-M, Rustagi A, Roque J, Asuni A, et al. Cytokine profile in plasma of severe COVID-19 does not differ from ARDS and sepsis. JCI Insight 2020;5. https://doi.org/10.1172/jci.insight.140289.

[8] Reis ES, Mastellos DC, Hajishengallis G, Lambris JD. New insights into the immune functions of complement. Nature Reviews Immunology 2019;19:503-16. https:// doi.org/10.1038/s41577-019-0168-x.

[9] Dunkelberger JR, Song W-C. Complement and its role in innate and adaptive immune responses. Cell Research 2010;20:34-50. https://doi.org/10.1038/ cr.2009.139.

[10] Ma L, Sahu SK, Cano M, Kuppuswamy V, Bajwa J, McPhatter J, et al. Increased complement activation is a distinctive feature of severe SARS-CoV-2 infection. Science Immunology 2021;6:eabh2259. https://doi.org/10.1126/sciimmunol.abh2259.

[11] Java A, Apicelli AJ, Liszewski MK, Coler-Reilly A, Atkinson JP, Kim AH, et al. The complement system in COVID-19: friend and foe? JCI Insight 2020;5:e140711. https://doi.org/10.1172/jci.insight.140711.

[12] Risitano AM, Mastellos DC, Huber-Lang M, Yancopoulou D, Garlanda C, Ciceri F, et al. Complement as a target in COVID-19? Nature Reviews Immunology 2020;20:343-4. https://doi.org/10.1038/s41577-020-0320-7.

[13] Magro C, Mulvey JJ, Berlin D, Nuovo G, Salvatore S, Harp J, et al. Complement associated microvascular injury and thrombosis in the pathogenesis of severe COVID19 infection: A report of five cases. Translational Research : The Journal of Laboratory and Clinical Medicine 2020;220:1-13. https://doi.org/10.1016/j. trsl.2020.04.007.

[14] Yu J, Yuan X, Chen H, Chaturvedi S, Braunstein EM, Brodsky RA. Direct activation of the alternative complement pathway by SARS-CoV-2 spike proteins is blocked by factor D inhibition. Blood 2020;136:2080-9. https://doi.org/10.1182/ blood.2020008248.

[15] Holter JC, Pischke SE, de Boer E, Lind A, Jenum S, Holten AR, et al. Systemic complement activation is associated with respiratory failure in COVID-19 hospitalized 
patients. Proceedings of the National Academy of Sciences 2020;117:25018 https://doi.org/10.1073/pnas.2010540117.

[16] Li Q, Chen Z. An update: the emerging evidence of complement involvement in COVID-19. Medical Microbiology and Immunology 2021;210:101-9. https://doi. org/10.1007/s00430-021-00704-7.

[17] Carvelli J, Demaria O, Vély F, Batista L, Chouaki Benmansour N, Fares J, et al. Association of COVID-19 inflammation with activation of the C5a-C5aR1 axis. Nature 2020;588:146-50. https://doi.org/10.1038/s41586-020-2600-6.

18] Zelek WM, Cole J, Ponsford MJ, Harrison RA, Schroeder BE, Webb N, et al. Complement Inhibition with the C5 Blocker LFG316 in Severe COVID-19. American Journal of Respiratory and Critical Care Medicine 2020;202:1304-8. https://doi.org 10.1164/rccm.202007-2778LE.

[19] Annane D, Heming N, Grimaldi-Bensouda L, Frémeaux-Bacchi V, Vigan M, Roux A$\mathrm{L}$, et al. Eculizumab as an emergency treatment for adult patients with severe COVID-19 in the intensive care unit: A proof-of-concept study. EClinicalMedicine 2020;28. https://doi.org/10.1016/j.eclinm.2020.100590.

[20] Vlaar APJ, de Bruin S, Busch M, Timmermans SAMEG, van Zeggeren IE, Koning R, et al. Anti-C5a antibody IFX-1 (vilobelimab) treatment versus best supportive care for patients with severe COVID-19 (PANAMO): an exploratory, open-label, phase 2 randomised controlled trial. The Lancet Rheumatology 2020;2:e764-73. https://doi.org/10.1016/S2665-9913(20)30341-6.

[21] Mastaglio S, Ruggeri A, Risitano AM, Angelillo P, Yancopoulou D, Mastellos DC et al. The first case of COVID-19 treated with the complement C3 inhibitor AMY101. Clinical Immunology (Orlando, Fla) 2020;215:108450. https://doi.org 10.1016/j.clim.2020.108450.

[22] Ackermann M, Anders H-J, Bilyy R, Bowlin GL, Daniel C, de Lorenzo R, et al. Patients with COVID-19: in the dark-NETs of neutrophils. Cell Death \& Differentiation 2021. https://doi.org/10.1038/s41418-021-00805-z.

[23] Veras FP, Pontelli MC, Silva CM, Toller-Kawahisa JE, de Lima M, Nascimento DC et al. SARS-CoV-2-triggered neutrophil extracellular traps mediate COVID-19 pathologySARS-CoV-2 directly triggers ACE-dependent NETs. Journal of Experimental Medicine 2020;217. https://doi.org/10.1084/jem.20201129.

[24] Zuo Y, Yalavarthi S, Shi H, Gockman K, Zuo M, Madison JA, et al. Neutrophil extracellular traps in COVID-19. JCI Insight 2020:5. https://doi.org/10.1172/jci.insight138999.

[25] Reusch N, de Domenico E, Bonaguro L, Schulte-Schrepping J, Baßler K, Schultze JL, et al. Neutrophils in COVID-19. Frontiers in Immunology 2021;12:652470. https://doi.org/10.3389/fimmu.2021.652470.

[26] Hazeldine J, Lord JM. Neutrophils and COVID-19: Active Participants and Rational Therapeutic Targets. Frontiers in Immunology 2021;12:2097.

[27] Meizlish ML, Pine AB, Bishai JD, Goshua G, Nadelmann ER, Simonov M, et al. A neutrophil activation signature predicts critical illness and mortality in COVID19. Blood Advances 2021;5:1164-77. https://doi.org/10.1182/bloodadvances.2020003568.

[28] Lévy Y, Wiedemann A, Hejblum BP, Durand M, Lefebvre C, Surénaud M, et al. CD177, a specific marker of neutrophil activation, is associated with coronavirus disease 2019 severity and death. IScience 2021;24:102711. https://doi.org/ 10.1016/j.isci.2021.102711.

[29] Price PJR, Bánki Z, Scheideler A, Stoiber H, Verschoor A, Sutter G, et al. Complement Component C5 Recruits Neutrophils in the Absence of C3 during Respiratory Infection with Modified Vaccinia Virus Ankara. The Journal of Immunology 2015;194:1164. https://doi.org/10.4049/jimmunol.1301410.

[30] Vandendriessche S, Cambier S, Proost P, Marques PE. Complement Receptors and Their Role in Leukocyte Recruitment and Phagocytosis. Frontiers in Cell and Developmental Biology 2021;9:144.

[31] Strich JR, Ramos-Benitez MJ, Randazzo D, Stein SR, Babyak A, Davey RT, et al. Fostamatinib Inhibits Neutrophils Extracellular Traps Induced by COVID-19 Patient Plasma: A Potential Therapeutic. The Journal of Infectious Diseases 2021;223:981-4. https://doi.org/10.1093/infdis/jiaa789.

[32] Delaveris CS, Wilk AJ, Riley NM, Stark JC, Yang SS, Rogers AJ, et al. Synthetic Siglec-9 Agonists Inhibit Neutrophil Activation Associated with COVID-19. ACS Central Science 2021;7:650-7. https://doi.org/10.1021/acscentsci.0c01669.

[33] Silvin A, Chapuis N, Dunsmore G, Goubet A-G, Dubuisson A, Derosa L, et al. Elevated Calprotectin and Abnormal Myeloid Cell Subsets Discriminate Severe from Mild COVID-19. Cell 2020;182:1401-18. https://doi.org/10.1016/j.cell.2020.08.002. .e18.

[34] Mahler M, Meroni P-L, Infantino M, Buhler KA, Fritzler MJ. Circulating Calprotec tin as a Biomarker of COVID-19 Severity. Expert Review of Clinical Immunology 2021;17:431-43. https://doi.org/10.1080/1744666X.2021.1905526.
[35] Guo Q, Zhao Y, Li J, Liu J, Yang X, Guo X, et al. Induction of alarmin S100A8/A9 mediates activation of aberrant neutrophils in the pathogenesis of COVID-19. Cell Host \& Microbe 2021;29:222-35. https://doi.org/10.1016/j.chom.2020.12.016. e4

[36] Pacha O, Sallman MA, Evans SE. COVID-19: a case for inhibiting IL-17? Nature Reviews Immunology 2020;20:345-6. https://doi.org/10.1038/s41577-0200328-z.

[37] Shibabaw T. Inflammatory Cytokine: IL-17A Signaling Pathway in Patients Present with COVID-19 and Current Treatment Strategy. Journal of Inflammation Research 2020;13:673-80. https://doi.org/10.2147/JIR.S278335.

[38] Wu D, Yang XO. TH17 responses in cytokine storm of COVID-19: An emerging target of JAK2 inhibitor Fedratinib. Journal of Microbiology, Immunology and Infection 2020;53:368-70. https://doi.org/10.1016/j.jmii.2020.03.005.

[39] Zhang B, Yin Y, Lai RC, Tan SS, Choo ABH, Lim SK. Mesenchymal Stem Cells Secrete Immunologically Active Exosomes. Stem Cells and Development 2013;23:123344. https://doi.org/10.1089/scd.2013.0479.

[40] Robinson PJ. Differential stimulation of protein kinase $C$ activity by phorbol ester or calcium/phosphatidylserine in vitro and in intact synaptosomes. Journal of Biological Chemistry 1992;267:21637-44. https://doi.org/10.1016/S0021-9258(19) 36659-1.

[41] Lai RC, Yeo RWY, Tan SS, Zhang B, Yin Y, Sze NSK, et al. Mesenchymal stem cell exosomes: the future MSC-based therapy? Mesenchymal stem cell therapy 2013: 39-61. Springer

[42] Noris M, Benigni A, Remuzzi G. The case of complement activation in COVID-19 multiorgan impact. Kidney International 2020;98:314-22. https://doi.org/ 10.1016/j.kint.2020.05.013

[43] Ye W, Chen G, Li X, Lan X, Ji C, Hou M, et al. Dynamic changes of D-dimer and neutrophil-lymphocyte count ratio as prognostic biomarkers in COVID-19. Respiratory Research 2020;21:169. https://doi.org/10.1186/s12931-020-01428-7.

[44] Middleton EA, He X-Y, Denorme F, Campbell RA, Ng D, Salvatore SP, et al. Neutrophil extracellular traps contribute to immunothrombosis in COVID-19 acute respiratory distress syndrome. Blood 2020;136:1169-79. https://doi.org/ 10.1182/blood.2020007008.

[45] Skendros P, Mitsios A, Chrysanthopoulou A, Mastellos DC, Metallidis S, Rafailidis $P$, et al. Complement and tissue factor-enriched neutrophil extracellular traps are key drivers in COVID-19 immunothrombosis. The Journal of Clinical Investigation 2020;130:6151-7. https://doi.org/10.1172/JCI141374.

[46] Mutua V, Gershwin LJ. A Review of Neutrophil Extracellular Traps (NETs) in Disease: Potential Anti-NETs Therapeutics. Clinical Reviews in Allergy \& Immunology 2021;61:194-211. https://doi.org/10.1007/s12016-020-08804-7.

[47] Kaplan MJ, Radic M. Neutrophil extracellular traps: double-edged swords of innate immunity. Journal of Immunology (Baltimore, Md : 1950) 2012;189:268995. https://doi.org/10.4049/jimmunol.1201719.

[48] Toh WS, Lai RC, Zhang B, Lim SK. MSC exosome works through a protein-based mechanism of action. Biochemical Society Transactions 2018;46:843-53. https:// doi.org/10.1042/BST20180079.

[49] Chen TS, Arslan F, Yin Y, Tan SS, Lai RC, Choo ABH, et al. Enabling a robust scalable manufacturing process for therapeutic exosomes through oncogenic immortalization of human ESC-derived MSCs. Journal of Translational Medicine 2011;9:47. https://doi.org/10.1186/1479-5876-9-47.

[50] Lai RC, Arslan F, Lee MM, Sze NSK, Choo A, Chen TS, et al. Exosome secreted by MSC reduces myocardial ischemia/reperfusion injury. Stem Cell Research 2010;4:214-22.

[51] Lai RC, Arslan F, Tan SS, Tan B, Choo A, Lee MM, et al. Derivation and characterization of human fetal MSCs: an alternative cell source for large-scale production of cardioprotective microparticles. Journal of Molecular and Cellular Cardiology 2010;48:1215-24

[52] Sze SK, de Kleijn DP v, Lai RC, Tan EKW, Zhao H, Yeo KS, et al. Elucidating the secretion proteome of human embryonic stem cell-derived mesenchymal stem cells. Molecular \& Cellular Proteomics 2007;6:1680-9.

[53] Zhang S, Chu WC, Lai RC, Lim SK, Hui JHP, Toh WS. Exosomes derived from human embryonic mesenchymal stem cells promote osteochondral regeneration. Osteoarthritis and Cartilage 2016;24(12):2135-40. https://doi.org/10.1016/j. joca.2016.06.022.

[54] Accarie A, I'Homme B, Benadjaoud MA, Lim SK, Guha C, Benderitter M, et al. Extracellular vesicles derived from mesenchymal stromal cells mitigate intestinal toxicity in a mouse model of acute radiation syndrome. Stem Cell Research \& Therapy 2020;371. (2020). https://doi.org/10.1186/s13287-020-01887-1. 